\title{
BMJ Open Perceived physical health in obsessive- compulsive disorder: a protocol for a systematic review and meta-analysis
}

\author{
Andrea Pozza, ${ }^{\oplus}$ Fabio Ferretti, Anna Coluccia
}

To cite: Pozza A, Ferretti F, Coluccia A. Perceived physical health in obsessivecompulsive disorder: a protocol for a systematic review and meta-analysis. BMJ Open 2019:9:e026261. doi:10.1136/ bmjopen-2018-026261

- Prepublication history for this paper is available online. To view these files, please visit the journal online (http://dx.doi. org/10.1136/bmjopen-2018026261).

Received 27 August 2018 Revised 18 March 2019 Accepted 14 May 2019
Check for updates

(C) Author(s) (or their employer(s)) 2019. Re-use permitted under CC BY-NC. No commercial re-use. See rights and permissions. Published by BMJ.

Department of Medical Sciences, Surgery and Neurosciences, Santa Maria alle Scotte University Hospital, Siena, Italy

Correspondence to Professor Fabio Ferretti; ferrefa@unisi.it

\section{ABSTRACT}

Introduction Obsessive-compulsive disorder (OCD) is a mental health condition associated with severe impairment in a variety of quality of life domains, an increased physical health burden, and a higher risk of general medical conditions and mortality compared with the general population. While there is a large amount of literature on psychological quality of life, a systematic review of perceived physical health in OCD is lacking. A quantitative summary might suggest that policy makers also focus the evaluation on perceived physical health and develop new strategies also aimed at this outcome. The current paper presents a protocol for the first systematic review and meta-analysis aimed at summarising perceived physical health in OCD by specifically examining perceived physical health status, bodily pain and role limitations due to physical problems in patients with OCD compared with controls. The review will also investigate potential demographic and clinical moderators of perceived physical health status (age, gender, OCD severity, publication date, methodological quality).

Methods and analysis A systematic review and meta-analysis will be conducted according to PRISMA guidelines. Studies will be included if using a clinical group with a current primary diagnosis of OCD established by international standardised criteria, if measuring perceived physical health status, and if using screened or community participants as controls. No publication date or language restriction will be applied. An online systematic search of electronic databases (Scopus, PubMed, PsycINF0, EMBASE, Cochrane Library), and examination of conference proceedings and theses/dissertations will be conducted by independent reviewers. Risk of bias will be assessed through the Newcastle-Ottawa Scale.

Ethics and dissemination The current review does not require ethics approval. The results will be disseminated through publications in peer-reviewed journals.

PROSPERO registration number 2018 CRD42018106194.

\section{INTRODUCTION}

Obsessive-compulsive disorder (OCD) is a mental health condition which consists of unwanted, intrusive, anxiety-evoking thoughts, mental images or impulses, defined as obsessions, and repetitive behaviours or mental actions, defined as compulsions, engaged in by an individual to cope with the

\section{Strengths and limitations of the study}

- A strength of the review is the analysis of demographic, clinical and study-related moderators of perceived physical health.

- Another strength is the sensitivity analysis by which effect sizes are calculated for studies using screened healthy controls only.

- A further strength is that the search for and selection of studies are performed by two independent reviewers who will also perform a methodological quality assessment.

- A limitation is that lack of information might prevent analysis of comorbid general medical disease as a moderator.

- Another potential limitation concerns the use of self-report measures of perceived physical health.

anxiety provoked by the obsessions. ${ }^{1}$ This condition typically presents with a chronic or intermittent, often deteriorating, temporal course and affects approximately 1\%-2\% of the general population. ${ }^{1}$ Evidence reported by several studies shows that OCD is associated with severe impairment in a variety of quality of life domains, including psychological quality of life, social relationships, and family or work-related life. ${ }^{2-8}$ Severe impairment in psychological quality of life is present particularly in female patients and even in patients with mild-to-moderate symptom severity, since an inverse relationship has been found between OCD severity and quality of life. ${ }^{9}$

The physical domain of health is under-recognised by practitioners since intervention typically focuses on the purely mental/ psychological component of OCD, such as obsessions/compulsions or anxiety. ${ }^{10}$ There are several reasons why evaluating perceived physical health is important to advance research and clinical practice in OCD. First, the existing studies which investigated perceived physical health in OCD produced inconsistent findings: some data suggest that perceived physical health in OCD is not significantly poorer than in 
healthy or community controls, ${ }^{211}$ whereas other contributions provided evidence that in OCD there is a poorer perception of physical health than in controls. ${ }^{12-14}$ Second, according to some epidemiological studies, a large subgroup of patients, around $50 \%$, present with general medical conditions. ${ }^{10}$ Risk of developing general medical conditions in OCD has been found to be higher for female gender, older age, and longer duration of untreated illness. ${ }^{10}$ Thus, the focus of clinicians for this subgroup of patients should also be directed at targeting physical health in clinical practice. Third, prospective data suggest that OCD is also associated with an increased physical health burden and double the risk of mortality compared with the general population. ${ }^{15}$ This risk seems to remain high even after controlling for other comorbid psychiatric disorders such as depressive, anxiety or addictive disorders. ${ }^{15}$ Lastly, certain subtypes of OCD symptoms such as obsessions related to contamination and those linked to responsibility for harm may have a related physical health focus. ${ }^{16}{ }^{17}$ For example, compulsions can lead the person suffering from OCD to avoid social interactions or sports, or have unhealthy eating habits because of contamination fears towards certain types of food/ objects/places. In addition, it seems that there is a clinical overlap between OCD and some health anxiety maintenance behaviours such as cyberchondria, reassurance seeking and anxiety/disgust sensitivity. ${ }^{17-23}$ Specifically focusing on the perceived component of physical health specifically as opposed to other aspects of physical health is important because it is based on the WHO's definition of health as a state of complete physical, mental and social well-being and not merely the absence of disease or infirmity. ${ }^{24}$ In addition, specifically investigating perceived physical health in OCD specifically as opposed to other aspects of physical health is relevant because OCD is often characterised by features negatively affecting perception of physical health such as a selective attention to body and depression symptoms, present in about $50 \%$ of the patients. $^{2025-27}$

Therefore, it may be expected that patients with OCD are more likely to report negative perceived physical health in comparison with controls with no psychiatric disorders or with individuals recruited from the general population. The impact of OCD on perceived physical health might be associated with an unhealthy lifestyle caused by the symptoms. ${ }^{10}$ Some demographic factors, including age and gender, might be moderators of a poorer perceived physical health in OCD. The role of older age as a moderator might be hypothesised considering previous data which indicate that a higher risk of general medical conditions in OCD is associated with older age. ${ }^{10}$ Moreover, accumulating evidence suggests gender-based peculiarities in the clinical picture of OCD: it might be hypothesised that female gender is associated with worse perceived physical health since women more frequently report medical disorders, depression symptoms, contamination-based symptoms, suicidal ideation and comorbid eating disorders, which appear to be clinical variables potentially impacting the perception of physical health. ${ }^{28}$ OCD severity might be considered as a clinical variable moderating the impact of the disorder on physical health in the same manner as for psychological quality of life since less severe symptoms and higher severity were also found to be related to negative psychological quality of life. ${ }^{9}$

Starting from the abovementioned points, a clear understanding of perceived physical health in OCD appears necessary. Some indications suggest that OCD is associated with negative physical health outcomes and that physical health in OCD tends to be poorer than in healthy controls. Knowledge of which variables might be associated with poorer physical health can help clinical practice. In the current literature, no study has provided a quantitative summary of perceived physical health in OCD: a systematic review is lacking. Therefore, this paper presents the protocol for the first systematic review and meta-analysis study aimed at summarising the current cross-sectional evidence about perceived physical health in OCD, and specifically: (1) Perceived physical health status in patients with OCD compared with controls (screened healthy individuals or individuals from the general population). (2) Demographic, clinical and publication-related moderators of perceived physical health status (age, gender, OCD severity, publication date, methodological quality). (3) Additional outcomes related to perceived physical health status, including bodily pain and role limitations due to physical problems.

\section{METHODS}

\section{Eligibility criteria}

The protocol of the present systematic review is reported in accordance with the criteria of the Preferred Reporting Items for Systematic Review and Meta-Analysis Proto$\mathrm{cols}^{29}$ and was registered in the International prospective register of systematic reviews (PROSPERO) (2018 CRD42018106194). Any amendments will be updated on PROSPERO and documented accordingly. Eligibility criteria for the studies to be included involved the following domains: (A) Characteristics of participants. (B) Characteristics of outcomes. (C) Characteristics of comparators. (D) Characteristics of design.

\section{Characteristics of participants}

Studies will be included if they were conducted on a clinical group with a current primary diagnosis of OCD. Diagnosis shall have been established by using a semistructured clinical interview based on the criteria of a standardised diagnostic system such as the Structured Clinical Interview for the Diagnostic and Statistical Manual of Mental Disorders fourth edition (DSM-IV) ${ }^{30}$ or by using an unstructured clinical interview conducted by a mental health professional based on the criteria of a standardised diagnostic system such as the Diagnostic and Statistical Manual of Mental Disorders fifth edition $(\mathrm{DSM}-5)^{1}$ or the International Classification of Diseases 
and Related Health Problems tenth revision (ICD-10). ${ }^{31}$ Studies including patients with a lifetime diagnosis of OCD and studies using participants with subthreshold OCD will not be included. Studies that have used samples of any age (child, adolescent, adult and elderly participants) will be included. Studies using participants with primary hoarding will be excluded since this disorder is conceptualised as a separate diagnosis with a distinct clinical picture in the DSM-5. ${ }^{1}$ Studies will be included if they used outpatients, inpatients or patients recruited from day-hospital services, whether public or private. Studies will be included if they recruited patients from primary, secondary or tertiary healthcare contexts. A concurrent treatment, whether psychological or pharmacological, will not be considered as a reason for exclusion. Comorbid general medical disorders will not be a reason for exclusion.

\section{Characteristics of outcomes}

Studies will be included if they measured perceived physical health status, bodily pain or role limitations due to physical problems using validated, internationally recognised self-report instruments such as the Medical Outcomes Survey 36-Item Short-Form Health Survey (SF-36) ${ }^{32}$ or the WHO Quality of Life-Brief Form (WHOQOL-BREF). ${ }^{33}$ Measures of any length will be eligible for inclusion. As we are interested in investigating perceived physical health as a global construct, measures will be included if they assess physical health status, bodily pain or role limitations due to physical problems as global constructs. Measures will be excluded if they assess a specific aspect of perceived physical health, such as perceived physical health status related to specific body parts or functions (eg, perceived leg or muscle health/ pain).

\section{Characteristics of comparators}

Studies will be included if they used control groups of screened participants who did not meet the criteria for any psychiatric disorder included in a standardised classification system and this condition was verified during a clinical interview by a mental health practitioner. In addition, studies will be included if they used control groups of unscreened participants drawn from the general population.

\section{Characteristics of design}

Studies will be included if they used a cross-sectional case-control design where a group of patients with a primary OCD diagnosis was compared with a healthy or a general population control group on measures of perceived physical health. Other types of research will not be included unless the study provides the data necessary to calculate an effect size estimate (for the data needed to calculate the effect sizes, see 'Meta-analysis and summary measures'). No publication date or language restriction will be applied. Only cross-sectional case-control studies will be included as the aim of the review is to compare perceived physical health outcome of patients with OCD with control participants (ie, to investigate whether patients have impaired perceived physical health as compared with controls, whether that are healthy screened participants without the disorder or unscreened participants recruited from the general population).

\section{Search procedure}

Studies will be identified by conducting an online systematic search of electronic databases through a combination of Medical Subject Headings (MeSH) and keywords related to 'Obsessive Compulsive Disorder' and 'Perceived physical Health'. MeSH terms were created by using the PubMed MeSH on Demand tool which allowed us to identify relevant MeSH terms. The search procedure will be performed during the last week of March 2019 by using electronic databases Scopus, PubMed, Psychological Information Database (PsycINFO), Excerpta Medica dataBASE (EMBASE) and Cochrane Library. In order to ensure that the identified MeSH terms and keywords produced the studies we are aware of, two test searches were randomly extracted from all the planned searches and were conducted by a reviewer (AP) who entered "Obsessive Compulsive Disorder" AND "Perceived Physical Health" on PubMed, "Obsessive Compulsive Disorder" AND "Physical Quality of Life" on Scopus. This query produced some of the studies we had identified. ${ }^{211-14}$

Subsequently, the corresponding authors of the included studies will be contacted with a request for further data they might have. The reference lists of the studies included in the meta-analysis will also be examined. Conference proceedings will be hand-searched from inception to identify potential abstracts, papers or posters relevant to OCD presented to the following international scientific societies: American Psychiatry Association, American Psychological Association, Anxiety and Depression Association of America, Association for Behavioral and Cognitive Therapies, British Psychological Society, European Association of Psychology, European Psychiatry Association, International Obsessive Compulsive Disorder Foundation, Royal College of Psychiatrists. Eligible theses and doctoral dissertations will be identified by the two independent reviewers who will run the same queries on the open access theses and dissertations website. No restriction on publication and language will be applied. Details of the electronic search procedure are presented in table 1.

\section{Study selection process}

Studies will be assessed on eligibility criteria by two independent reviewers (AP, FF) at three different stages. In the first stage, studies will be screened by reading the title, in the second stage studies will be screened by reading the abstract. At both these stages, studies will be retained whether both reviewers agree or not on inclusion between the reviewers. Finally, retained studies will be assessed on eligibility criteria after reading the full text 
Table 1 Electronic search procedure

\section{Electronic databases}

Scopus (https://www.scopus.com)

PubMed (https://www.ncbi.nlm.nih.gov/pubmed)

PsycINFO (https://www.apa.org/pub/databases/psycinfo)

EMBASE (https://www.embase.com)

Cochrane Library (https://www.cochranelibrary.com)

Search terms

Medical Subject Headings (MeSH): "Obsessive Compulsive Disorder" AND "Physical Health"

Keywords:

"Obsessive Compulsive Disorder" AND "Physical Health", "Obsessive Compulsive Disorder" AND "Physical Health Status", Obsessive Compulsive Disorder" AND "Physical Quality of Life", "Obsessions" AND "Physical Health", "Obsessions" AND "Physical Health Status", Obsessions" AND "Physical Quality of Life", "Compulsions" AND "Physical Health", "Compulsions" AND "Physical Health Status", "Compulsions" AND "Physical Quality of Life".

of the article. At the third stage of selection, the reviewers will discuss reasons for inclusion and any disagreement between them will be addressed during meetings with another independent reviewer (AC) in order to come to an agreement on the pool of studies to be included in the meta-analysis.

\section{Data extraction and coding}

All the information will be extracted from each of the included studies and inserted into an Excel worksheet by two independent reviewers (AP, FF). This process will be first piloted on two included studies randomly extracted from the total group of the included studies. The information which will be collected and coded from each of the studies is provided in table 2.

A third independent reviewer (AC) not involved in the first phase of the extraction process will verify and check the correctness of the data inserted by the two reviewers in the worksheet. After data insertion is completed, discrepancies in the data extracted by the two reviewers will be discussed and resolved in a final meeting between the data extraction reviewers and the third independent reviewer.

\section{Moderators coding}

Since large and significant effect size inconsistency is expected, moderators will be examined. Two independent reviewers (FF and AP) will code the moderators. During subsequent meetings between the two reviewers, insertion of the data in the worksheet will be checked for accuracy and any discrepancies will be discussed and resolved with a third reviewer (AC). The following variables will be coded as moderators: (A) Mean age of the sample. (B) Gender of the sample (coded as the percentage of female participants). (C) OCD symptom severity, coded as a continuous variable based on the scores on the Yale-Brown Obsessive Compulsive Scale, ${ }^{34}$ the gold standard measure for OCD severity assessment. (D) Publication date of the study. (E) Methodological quality of the studies, coded in terms of the scores on the Newcastle-Ottawa Scale $(\mathrm{NOS})^{35}$ (see 'Quality assessment').

\section{Quality assessment}

Quality of the included studies will be examined by adopting the NOS. This tool has been recently recommended by systematic review practice guidelines as the most reliable instrument for conducting quality assessment of cross-sectional or cohort studies in systematic reviews. ${ }^{36}$ The NOS includes eight items grouped into three key domains: (1) Selection. (2) Comparability. (3) Exposure (case-control studies) according to the study design. For each item a series of response options is provided. A star system permits semiquantitative quality assessment. At most, the highest quality studies receive one star per item, except comparability where two stars may be assigned. Total NOS Scores range from 0 to 9 stars. Two independent reviewers (AP, FF) will conduct the methodological quality assessment. Discrepancies in the assignment of the scores will be resolved by a consensus meeting with a third independent reviewer (AC).

\section{Meta-analysis and summary measures}

The meta-analysis will be conducted by using the random-effects models which assume that the included studies are drawn from populations of studies that systematically differ from each other. ${ }^{34}$ According to these models, the effect sizes obtained from primary studies differ due to random errors within studies (as in the fixed-effect model), and because of true variation in effect sizes from one study to the other. ${ }^{37}$ The effect sizes will be calculated as standardised mean differences (SMDs) using the following formula by Cohen ${ }^{38}:\left(M_{O C D}\right.$ $\left.-M_{\text {CONTROL }}\right) / S D_{\text {POOLED }}$, where $M_{\text {OCD }}$ represents the mean of the clinical groups of patients with OCD on the perceived physical health status measure (or the measure of role limitations due to physical problems or the measure of bodily pain), $M_{\text {CONTROL }}$ indicates the mean of the controls on those measures and $S D_{\text {POOLED }}$ is the pooled SD. The 
Table 2 Extraction and coding of information from the primary studies

\section{Information extracted}

Coding
1. Title of the paper
2. First author name
3. Publication date
4. Country where the study was conducted

5. Inclusion and exclusion criteria

\section{Total sample size}

7. Patients with OCD

8. Controls

\section{Types of controls}

\section{Perceived physical health status outcome in the OCD} group

11. Perceived physical health status outcome in the control group

12. Role limitations due to physical problems in the OCD group

13. Role limitations due to physical problems in the control group

\section{Bodily pain in the OCD group}

15. Bodily pain in the control group

16. Age of participants in the study

17. Women in the study

18. Measure(s) used to assess perceived physical health status Acronym(s) of the measure(s) used to assess perceived

19. Measure(s) used to assess role limitations due to physical problems

20. Measure(s) to assess bodily pain

21. OCD symptom severity

22. Research design

23. Instrument(s) used to establish the OCD diagnosis

24. Patients taking medication

25. Strategies used to recruit patients

\section{Strategies used to recruit controls}

27. Patients with comorbid personality disorders

28. Patients with comorbid general medical diseases

29. Patients with comorbid anxiety disorders

30. Patients with comorbid depressive disorders

31. Measure(s) used to assess anxiety symptoms

32. Measure(s) used to assess depression symptoms physical health status

Quote the statements in the paper describing participants inclusion/exclusion criteria

Total sample size

Number of patients with OCD

Number of controls

Screened healthy participants without psychiatric disorders or unscreened participants recruited from the general population

Mean and SD of the OCD group on the perceived physical health status outcome

Mean and SD of the control group on the perceived physical health status outcome

Mean and SD of the OCD group on the measure of role imitations due to physical problems

Mean and SD of the control group on the measure of role limitations due to physical problems

Mean and SD of the OCD group on the measure of bodily pain Mean and SD of the control group on the measure of bodily pain

Total mean age and age range

Total percentage of women

Acronym(s) of the measure(s) used to assess role limitations due to physical problems

Acronym(s) of the measure(s) used to assess bodily pain

Y-BOCS Scores

Case-control or prospective study

Acronym(s) of the instrument(s) used to establish the OCD diagnosis

Percentage of patients taking medication

Quote the statement in the paper describing the strategies used to recruit patients

Quote the statement in the paper describing the strategies used to recruit controls

Percentage of patients with comorbid personality disorders Percentage of patients with comorbid general medical diseases

Percentage of patients with comorbid anxiety disorders Percentage of patients with comorbid depressive disorders

Acronym(s) of the measure(s) used to assess anxiety symptoms

Acronym(s) of the measure(s) used to assess depression symptoms

OCD, obsessive-compulsive disorder; Y-BOCS, Yale-Brown Obsessive Compulsive Scale. 
effect sizes will be estimated by using $95 \%$ CI and interpreted according to the criteria described by Cohen: ${ }^{38}$ values equal to or higher than 0.80 are interpreted as large, values up to 0.50 as moderate, and values up to 0.20 as small. Negative effect sizes indicate poorer perceived physical health in patients with OCD than in controls. When a study reports data on more than one measure of physical health status such as on both the SF-36 Physical Health Status and the WHOQOL-BREF Physical Health Status Scales, a mean effect size will be computed by pooling the effect sizes obtained from all the measures of physical health status. SMD will also be calculated separately for the data obtained from role limitations due to the state of physical problems to summarise the evidence on the perceived negative interference of physical health-related problems. Finally, SMD will also be calculated separately for the data obtained from bodily pain scales to summarise the data related to the perception of physical pain in OCD.

To verify publication bias, three different procedures have been chosen, including Duval and Tweedie's trim and fill procedure, ${ }^{39}$ visual inspection of the funnel plot and Egger's test.

Sensitivity analyses will be conducted by calculating the effect sizes only in the subgroups of studies (1) Using SF-36 to measure perceived physical health status. (2) Including only adult participants. (3) Including only screened healthy individuals as controls.

To assess between-studies heterogeneity, two complementary indices will be computed, the $I^{2}$ index ${ }^{40}$ and the $Q$ statistic, ${ }^{41}$ respectively. The $I^{2}$ index is a measure of between-study heterogeneity expressed as a percentage, which is attributable to variability rather than chance. ${ }^{40}$ A value close to 0 indicates homogeneity, whereas values of $25 \%-50 \%, 50 \%-75 \%$ and $75 \%-100 \%$ indicate low, moderate and high heterogeneity, respectively. The $Q$ index is computed by summing the squared deviations of each study's effect estimate from the overall effect estimate while weighting the contribution of each study by its inverse variance. ${ }^{42}$ In the case of homogeneity among the effect sizes, the $Q$ statistic follows a $\chi^{2}$ distribution with $\mathrm{k}-1 \mathrm{df}$, $\mathrm{k}$ being the number of studies. Moderator's analysis will be conducted by performing weighted least square meta-regressions. The meta-analysis will be conducted by using the Comprehensive Meta-analysis Software program V.2.00.

\section{Patient and public involvement}

Patients and the public were not involved in the development phase of the research question, of the outcome measures, and of the systematic review and meta-analysis protocol. The study does not involve patient recruitment, and patients were not involved in conduction of the study.

\section{DISCUSSION AND CONCLUSIONS}

While evidence of impairment of psychological quality of life in OCD is well established, ${ }^{3-5}$ the studies on perceived physical health have not provided consistent evidence. In some studies patients with OCD reported a similar perception of physical health to controls, whereas in other studies, physical health in patients with OCD was lower than controls. The current manuscript describes the first systematic review and meta-analysis study aimed at providing a quantitative summary of current evidence on a variety of perceived physical health outcomes in OCD as compared with controls, including physical health status, bodily pain and role limitations due to physical problems. Finally, demographic (age and gender), clinical (OCD severity) and study-related moderators (publication date, methodological quality) will be investigated. The main methodological strengths of this review are the sensitivity analyses of studies conducted only on screened healthy controls, the fact that review decisions are made by two independent reviewers, and methodological quality assessment.

The physical domain of health is not sufficiently considered by researchers and clinicians working with OCD: intervention typically focuses on the purely mental/ psychological component of OCD such as obsessions/ compulsions or anxiety. ${ }^{10}$ A quantitative synthesis of perception of physical health in OCD may have important clinical and research implications. Physical health might be considered a target of treatment as an increase in physical health could be expected to modulate improvement in OCD symptoms. Consistent with this hypothesis, some recent research indicated that health-focused interventions and aerobic exercise as adjunct to standard treatment were effective in reducing OCD symptoms and secondary outcomes such as anxiety and depression. ${ }^{434}$ Moreover, certain treatment approaches such as mindfulness-based or group interventions might be coadjuvant strategies for patients with OCD to help them develop a non-judgemental attitude and reduce their concentrated focus on thoughts/inner experiences and to open up to a perception of body and also to positive feelings. ${ }^{45} 46$

Contributors AP and FF conceived and designed the study and wrote the first draft of the paper. AC conceived and designed the study and critically reviewed the first draft of the paper.

Funding The authors have not declared a specific grant for this research from any funding agency in the public, commercial or not-for-profit sectors.

Competing interests None declared.

Patient consent for publication Not required.

Provenance and peer review Not commissioned; externally peer reviewed.

Open access This is an open access article distributed in accordance with the Creative Commons Attribution Non Commercial (CC BY-NC 4.0) license, which permits others to distribute, remix, adapt, build upon this work non-commercially, and license their derivative works on different terms, provided the original work is properly cited, appropriate credit is given, any changes made indicated, and the use is non-commercial. See: http://creativecommons.org/licenses/by-nc/4.0/.

\section{REFERENCES}

1. American Psychiatric Association,. Diagnostic and Statistical Manual of Mental Disorders (DSM5). $5^{\text {th }}$ ed. Washington, DC: American Psychiatric Association, 2013. 
2. Albert U, Maina G, Bogetto F, et al. Clinical predictors of healthrelated quality of life in obsessive-compulsive disorder. Compr Psychiatry 2010;51:193-200.

3. Coluccia A, Fagiolini A, Ferretti F, et al. Adult obsessive-compulsive disorder and quality of life outcomes: A systematic review and metaanalysis. Asian J Psychiatr 2016;22:41-52.

4. Coluccia A, Fagiolini A, Ferretti F, et al. Obsessive-Compulsive Disorder and quality of life outcomes: protocol for a systematic review and meta-analysis of cross-sectional case-control studies. Epidemiology, Biostatistics and Public Health 2015;12:2.

5. Coluccia A, Ferretti F, Fagiolini A, et al. Quality of life in children and adolescents with obsessive-compulsive disorder: a systematic review and meta-analysis. Neuropsychiatr Dis Treat 2017;13:597-608.

6. Lochner C, Mogotsi M, du Toit PL, et al. Quality of life in anxiety disorders: a comparison of obsessive-compulsive disorder, social anxiety disorder, and panic disorder. Psychopathology 2003;36:255-62.

7. Remmerswaal KCP, Batelaan NM, Smit JH, et al. Quality of life and relationship satisfaction of patients with Obsessive Compulsive Disorder. J Obsessive Compuls Relat Disord 2016;11:56-62.

8. Subramaniam M, Soh P, Ong C, et al. Patient-reported outcomes in obsessive-compulsive disorder. Dialogues Clin Neurosci 2014;16:239-54.

9. Pozza A, Lochner C, Ferretti F, et al. Does higher severity really correlate with a worse quality of life in obsessive-compulsive disorder? A meta-regression. Neuropsychiatr Dis Treat 2018;14:1013-23.

10. Aguglia A, Signorelli MS, Albert U, et al. The Impact of General Medical Conditions in Obsessive-Compulsive Disorder. Psychiatry Investig 2018;15:246-53.

11. Eisen JL, Mancebo MA, Pinto A, et al. Impact of obsessivecompulsive disorder on quality of life. Compr Psychiatry 2006;47:270-5.

12. Fontenelle IS, Fontenelle LF, Borges MC, et al. Quality of life and symptom dimensions of patients with obsessive-compulsive disorder. Psychiatry Research 2010;79:198-203.

13. Kumar A, Sharma MP, Kandavel T, et al. Cognitive appraisals and quality of life in patients with obsessive compulsive disorder. $J$ Obsessive Compuls Relat Disord 2012;1:301-5.

14. Srivastava S, Bhatia MS, Thawani R, et al. Quality of life in patients with obsessive compulsive disorder: A longitudinal study from India. Asian J Psychiatr 2011;4:178-82.

15. Meier SM, Mattheisen M, Mors O, et al. Mortality Among Persons With Obsessive-Compulsive Disorder in Denmark. JAMA Psychiatry 2016;73:268-74.

16. Fergus TA. The Cyberchondria Severity Scale (CSS): an examination of structure and relations with health anxiety in a community sample. J Anxiety Disord 2014;28:504-10.

17. Pozza A, Dèttore D. Are inflated responsibility beliefs specific to OCD? meta-analysis of the relations of responsibility to OCD, anxiety disorders, and depression symptoms. Clinical Neuropsychiatry 2014;11:170-81.

18. Fergus TA, Russell LH. Does cyberchondria overlap with health anxiety and obsessive-compulsive symptoms? An examination of latent structure and scale interrelations. J Anxiety Disord 2016;38:88-94.

19. Norr AM, Oglesby ME, Raines AM, et al. Relationships between cyberchondria and obsessive-compulsive symptom dimensions. Psychiatry Res 2015;230:441-6.

20. Raines AM, Oglesby ME, Capron DW, et al. Obsessive compulsive disorder and anxiety sensitivity: Identification of specific relations among symptom dimensions. J Obsessive Compuls Relat Disord 2014;3:71-6.

21. Solem S, Borgejordet S, Haseth S, et al. Symptoms of health anxiety in obsessive-compulsive disorder: Relationship with treatment outcome and metacognition. J Obsessive Compuls Relat Disord 2015;5:76-81.

22. Wright KD, Lebell MA, Carleton RN. Intolerance of uncertainty, anxiety sensitivity, health anxiety, and anxiety disorder symptoms in youth. J Anxiety Disord 2016;41:35-42.

23. Pozza A, Mazzoni GP, Berardi D, et al. Preliminary study on the psychometric properties of the Italian version of the Disgust Propensity and Sensitivity Scale-Revised (DPSS-R) in non-clinical and clinical samples with Obsessive-Compulsive Disorder and Anxiety Disorders. Psicoterapia Cognitiva e Comportamentale 2016;22:271-96.

24. World Health Organization (WHO). Health is a state of complete physical, mental and social well being and not merely the absence of disease or infirmity. WHO Basic documents. 42nd ed. Geneva: World Health Organization, 1999.Crino R, Andrews G. Obsessivecompulsive disorder and axis I comorbidity. Journal of Anxiety Disorders 1996:37-46.

25. Kugler BB, Lewin AB, Phares V, et al. Quality of life in obsessivecompulsive disorder: the role of mediating variables. Psychiatry Res 2013;206:43-9.

26. Pozza A, Coradeschi D, Dèttore D. Do dysfunctional beliefs moderate the negative influence of comorbid severe depression on outcome of residential treatment for refractory OCD? A pilot study. Clinical Neuropsychiatry 2013;10:72-83.

27. Jaisoorya TS, Reddy YC, Srinath S, et al. Sex differences in Indian patients with obsessive-compulsive disorder. Compr Psychiatry 2009;50:70-5.

28. Shamseer L, Moher D, Clarke M, et al. Preferred reporting items for systematic review and meta-analysis protocols (PRISMA-P) 2015: elaboration and explanation. BMJ 2015;349:97647.

29. First MB, Gibbon M, Spitzer RL, et al. Structured Clinical Interview for DSM-IV Axis II personality disorders (SCID-II). Washington, DC: American Psychiatric Press, 1997.

30. World Health Organization. The ICD-10 classification of mental and behavioural disorders: diagnostic criteria for research, 1993.

31. Ware JE, Sherbourne CD. The MOS 36-item short-form health survey (SF-36). Medical Care 1992;1992:473-83.

32. Whoqol Group. Development of the World Health Organization WHOQOL-BREF quality of life assessment. The WHOQOL Group. Psychol Med 1998;28:551-8.

33. Goodman WK, Price LH, Rasmussen SA, et al. The Yale-Brown Obsessive Compulsive Scale. I. Development, use, and reliability. Arch Gen Psychiatry 1989;46:1006-11.

34. Wells G, Shea B, O'Connell D, et al. Newcastle-Ottawa quality assessment scale cohort studies, 2014. Retrieved from. http:// www. ohri. ca/programs/clinical_epidemiology/oxford. Asp. (19 Nov 2015).

35. Zeng X, Zhang Y, Kwong JS, et al. The methodological quality assessment tools for preclinical and clinical studies, systematic review and meta-analysis, and clinical practice guideline: a systematic review. J Evid Based Med 2015;8:2-10.

36. Borenstein M, Hedges LV, Higgins JPT, et al. Introduction to metaanalysis. Chichester: John Wiley \& Sons, 2009.

37. Cohen J. Statistical power analysis for the behavioral sciences. New York: Routledge, 1988.

38. Duval S, Tweedie R. Trim and fill: A simple funnel-plot-based method of testing and adjusting for publication bias in meta-analysis. Biometrics 2000;56:455-63.

39. Higgins JP, Thompson SG, Deeks JJ, et al. Measuring inconsistency in meta-analyses. BMJ 2003;327:557-60.

40. Lipsey MW, Wilson D. Practical meta-analysis. Thousand Oaks, CA: Sage, 2001.

41. Hedges LV. Distribution theory for glass's estimator of effect size and related estimators. Journal of Educational Statistics 1981;6:107-28.

42. Abrantes AM, Brown RA, Strong DR, et al. A pilot randomized controlled trial of aerobic exercise as an adjunct to OCD treatment. Gen Hosp Psychiatry 2017;49:51-5.

43. Rector NA, Richter MA, Lerman B, et al. A pilot test of the additive benefits of physical exercise to CBT for OCD. Cogn Behav Ther 2015;44:328-40.

44. Sarris J, Camfield D, Berk M. Complementary medicine, selfhelp, and lifestyle interventions for obsessive compulsive disorder (OCD) and the OCD spectrum: a systematic review. J Affect Disord 2012;138:213-21.

45. Pozza A, Andersson G, Dèttore D. What does best evidence tell us about the efficacy of group cognitive-behavioral therapy for obsessive-compulsive disorder? Protocol for a systematic review and meta-analysis. Psychol Res Behav Manag 2015;8:225.

46. Pozza A, Dèttore D. Drop-out and efficacy of group versus individual cognitive behavioural therapy: What works best for ObsessiveCompulsive Disorder? A systematic review and meta-analysis of direct comparisons. Psychiatry Res 2017;258:24-36. 